\title{
Tunneling transmission in two quantum wires coupled by a magnetically defined barrier
}

\author{
Wei-Dong Sheng \\ CCAST (World Laboratory) P.O. Box 8730, Beijing 100080, China and Institute of Semiconductors, \\ Academia Sinica, P.O. Box 912, Beijing 100083, China \\ Ben-Yuan $\mathrm{Gu}^{\mathrm{a})}$ \\ CCAST (World Laboratory) P.O. Box 8730, Beijing 100080, China and Institute of Physics, \\ Academia Sinica, P.O. Box 603, Beijing 100080, China ${ }^{\mathrm{b})}$ \\ Jian Wang \\ Department of Physics, the University of Hong Kong, Pokfulam Road, Hong Kong, China \\ Jian-Bai Xia \\ Institute of Semiconductors, Academia Sinica, P.O. Box 912, Beijing 100083, China
}

(Received 21 July 1997; accepted for publication 2 September 1997)

\begin{abstract}
A numerical analysis of an electron waveguide coupler based on two quantum wires coupled by a magnetically defined barrier is presented with the use of the scattering-matrix method. For different geometry parameters and magnetic fields, tunneling transmission spectrum is obtained as a function of the electron energy. Different from that of conventional electron waveguide couplers, the transmission spectrum of the magnetically coupled quantum wires does not have the symmetry with regard to those geometrically symmetrical ports. It was found that the magnetic field in the coupling region drastically enhances the coupling between the two quantum wires for one specific input port while it weakens the coupling for the other input port. The results can be well understood by the formation of the edge states in the magnetically defined barrier region. Thus, whether these edge states couple or decouple to the electronic propagation modes in the two quantum wires, strongly depend on the relative moving directions of electrons in the propagating mode in the input port and the edge states in the magnetic region. This leads to a big difference in transmission coefficients between two quantum wires when injecting electrons via different input ports. Two important coupler specifications, the directivity and uniformity, are calculated which show that the system we considered behaves as a good quantum directional coupler. (c) 1997 American Institute of Physics.
\end{abstract} [S0021-8979(97)06223-3]

\section{INTRODUCTION}

Recent technological advances in nanometer-scale lithography and atomic-layer epitaxy, which can provide semiconductor microstructures smaller than the inelastic and elastic mean scattering lengths, have attracted much attention to the studies of mesoscopic systems, especially after the discovery of the quantized conductance phenomenon. ${ }^{1,2}$ Inspired by the prospect of building devices based on quantum interference effect, many authors have proposed various structures $^{3}$ which start from high mobility modulation-doped $\mathrm{Al}_{x} \mathrm{Ga}_{1-x} \mathrm{As} / \mathrm{GaAs}$ heterostructures. The most prominent advantage of quantum interference device lies in the fact that its operation is controlled by the relative phase of the electron waves and a very high switch speed can be achieved. The quantum transistor based on T-shaped electron waveguide proposed by Sols et al. ${ }^{4}$ has been fabricated recently. ${ }^{5}$

The electron waveguide couplers have been proposed by Alamo et al.$^{6,7}$ for the first time. The coupler consists of two infinitely long wires coupled by a potential barrier. In such a device, the transfer length at which a complete switch of the electron wave from one wire to another occurs is found of the same order of the electron phase coherent length. ${ }^{8}$

\footnotetext{
${ }^{a)}$ Electronic mail: guby@aphy01.iphy.ac.cn

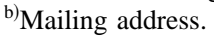

Macucci et al. ${ }^{9}$ also investigated 1D-to-1D tunneling in coupled electron waveguides. Recently, Vanbésien et al. ${ }^{10,11}$ proposed a structure for electron wave couplers. The structure consists of two electron waveguides coupled by an open interaction window instead of a tunneling barrier. Currently, the characteristics of electron transport in microstructures created by high mobility $\mathrm{Al}_{x} \mathrm{Ga}_{1-x} \mathrm{As} / \mathrm{GaAs}$ heterostructures in nonuniform magnetic fields have also become an extensive research subject of great theoretical and experimental interest. $^{12-18}$

To our knowledge, there are no studies on electron waveguide couplers in the presence of nonuniform magnetic fields theoretically or experimentally. In this article, we propose an electron waveguide coupler based on two quantum wires coupled by a magnetically defined barrier. It is schematically illustrated in Fig. 1. The coupler consists of two straight quantum wires and a coupling region, in which only the coupling region is subjected to a uniform magnetic field $B$ perpendicular to the plane of the coupler. Both quantum wires have the same width $W$ and are separated by a distance $L$. The coupling region has a width $D$. The device boundaries are defined by the hard-wall potentials which were found to be a good approximation in the single-mode regime. ${ }^{19}$ Here, we concentrate on the single-mode regime because the device operation in the fundamental transverse 


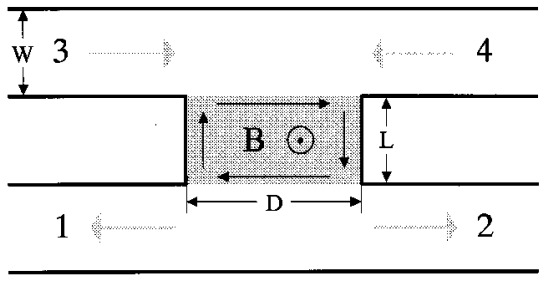

FIG. 1. Schematic illustration of magnetically coupled quantum wires, in which only the shadow area is subjected to a magnetic field.

mode is believed to be important for applications. ${ }^{4}$ We first present a numerical analysis of this coupler device. We then calculate tunneling transmission spectrum of the coupler for different geometric parameters and magnetic fields. We found that the transmission spectrum exhibits asymmetry with respect to geometrically symmetric ports. The magnetic field in the coupling region substantially enhances the coupling efficiency for one specific input port while it weakens the coupling for another input port. This character can be well interpreted by the formation of the edge states in the magnetically defined barrier, and the coupling or decoupling effect between the edge states and the propagating modes from different input ports. It is shown that this structure behaves as a good quantum directional coupler.

\section{SCATTERING APPROACH TO THE MAGNETICALLY COUPLED QUANTUM WIRES}

We employ a scattering approach to investigate transport properties of the magnetically coupled quantum wires as shown in Fig. 1. The transport properties of this four terminal device are determined by the overall scattering matrix. In order to obtain the overall scattering matrix, we divide the structure into three parts as shown in Fig. 2. Although the structure of the electron waveguide coupler is complicated, it is found that each individual unit is simple and easy to treat. Units $\mathrm{A}$ and $\mathrm{C}$ are $\mathrm{T}$-shaped structures with three ports, respectively. Similar structures have been studied in one of our early works. ${ }^{20}$ Unit B is a single magnetically defined barrier structure, which has been investigated by Takagaki and Ploog. ${ }^{14}$

We first build the scattering matrices for each individual unit, then construct the overall scattering matrix using the generalized composition law (see below), i.e.,

$$
\mathbf{S}=\mathbf{S}_{C} \otimes \mathbf{S}_{B} \otimes \mathbf{S}_{A}
$$

The scattering matrix is defined as follows: For a multiple-terminal scattering region, the wavefunctions in each terminal region can be expanded as

$$
\Psi=\sum_{n=1}^{M}\left(a_{n}^{+} e^{i k_{n}^{+} x}+a_{n}^{-} e^{-i k_{n}^{-} x}\right) \Phi_{n}(y),
$$

where $x$ and $y$ are the zonal longitudinal and transverse coordinates, respectively. $\Phi_{n}(y)$ is the wave function for the

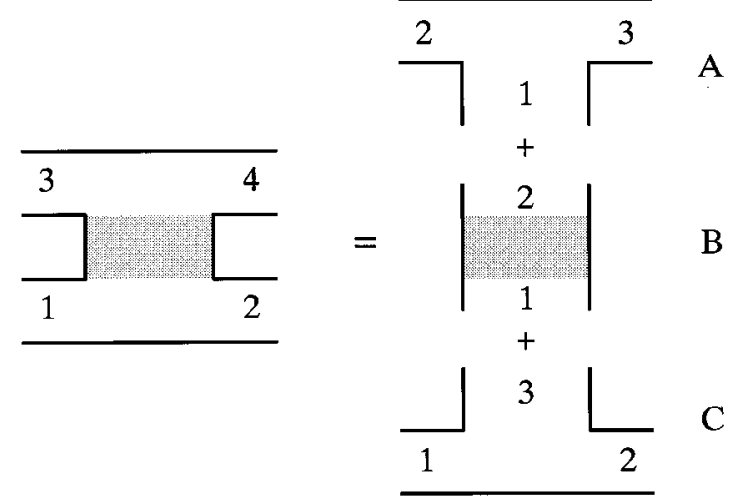

FIG. 2. Schematic illustration of three basic units divided from the device structure.

nth transverse mode, namely, $\sqrt{2 / W} \sin (n \pi y / W)$, in the absence of magnetic fields, where $W$ is the terminal width. $M$ is the number of transverse modes (including the evanescent modes). For the scattering region, we make use of a scattering matrix to relate the incoming and outgoing waves in all its ports. For unit A in Fig. 2, the scattering-matrix formulation is expressed as

$$
\left[\begin{array}{lll}
\mathbf{A}_{1}^{+} & \mathbf{A}_{2}^{-} & \mathbf{A}_{3}^{+}
\end{array}\right]^{T}=\mathbf{S}_{A}\left[\begin{array}{lll}
\mathbf{A}_{1}^{-} & \mathbf{A}_{2}^{+} & \mathbf{A}_{3}^{-}
\end{array}\right]^{T},
$$

where $\mathbf{A}^{ \pm}$consists of corresponding expansion coefficients $a_{n}^{ \pm}$. Similarly, for units B and C in Fig. 2, the scatteringmatrix formulations are given by

$$
\begin{aligned}
& {\left[\begin{array}{ll}
\mathbf{B}_{1}^{+} & \mathbf{B}_{2}^{-}
\end{array}\right]^{T}=\mathbf{S}_{B}\left[\begin{array}{ll}
\mathbf{B}_{1}^{-} & \mathbf{B}_{2}^{+}
\end{array}\right]^{T},} \\
& {\left[\begin{array}{lll}
\mathbf{C}_{1}^{-} & \mathbf{C}_{2}^{+} & \mathbf{C}_{3}^{-}
\end{array}\right]^{T}=\mathbf{S}_{C}\left[\begin{array}{lll}
\mathbf{C}_{1}^{+} & \mathbf{C}_{2}^{-} & \mathbf{C}_{3}^{+}
\end{array}\right]^{T} .}
\end{aligned}
$$

Because units $\mathrm{A}$ and $\mathrm{C}$ are not subjected to a magnetic field, we can employ the mode-matching method described in Ref. 20 to derive $\mathbf{S}_{A}$ and $\mathbf{S}_{C}$. For units $\mathrm{A}$ and $\mathrm{C}$, the number of modes in wide ports, e.g., port 1 for unit $\mathrm{A}$ and port 3 for unit $\mathrm{C}$, is taken different from that in other ports. The former is denoted by $N$ and the latter is denoted by $M$. Although the structures of units $\mathrm{A}$ and $\mathrm{C}$ are identical, their scattering matrices are not identical because of their different indices of port. The resulting semi-analytical expression of $\mathbf{S}_{A}$ and $\mathbf{S}_{C}$ reads

$$
\begin{aligned}
\mathbf{S}_{A}= & {\left[\begin{array}{ccc}
\mathbf{K}^{\prime}-\mathbf{C}_{1}^{\prime} & \mathbf{A}^{\prime} & \mathbf{F}^{\prime} \mathbf{A}^{\prime} \\
\mathbf{A} & \mathbf{K}-\mathbf{C}_{1} & \mathbf{C}_{2} \\
\mathbf{A} \mathbf{F}^{\prime} & \mathbf{C}_{2} & \mathbf{K}-\mathbf{C}_{1}
\end{array}\right]^{-1} } \\
& \times\left[\begin{array}{ccc}
\mathbf{K}^{\prime}+\mathbf{C}_{1}^{\prime} & -\mathbf{A}^{\prime} & -\mathbf{F}^{\prime} \mathbf{A}^{\prime} \\
-\mathbf{A} & \mathbf{K}+\mathbf{C}_{1} & -\mathbf{C}_{2} \\
-\mathbf{A} \mathbf{F}^{\prime} & -\mathbf{C}_{2} & \mathbf{K}+\mathbf{C}_{1}
\end{array}\right],
\end{aligned}
$$




$$
\begin{aligned}
\mathbf{S}_{C}= & {\left[\begin{array}{ccc}
\mathbf{K}-\mathbf{C}_{1} & \mathbf{C}_{2} & \mathbf{F A} \\
\mathbf{C}_{2} & \mathbf{K}-\mathbf{C}_{1} & \mathbf{F A F} \mathbf{F}^{\prime} \\
\mathbf{A}^{\prime} \mathbf{F} & \mathbf{F}^{\prime} \mathbf{A}^{\prime} \mathbf{F} & \mathbf{K}^{\prime}-\mathbf{C}_{1}^{\prime}
\end{array}\right]^{-1} } \\
& \times\left[\begin{array}{ccc}
\mathbf{K}+\mathbf{C}_{1} & -\mathbf{C}_{2} & -\mathbf{F A} \\
-\mathbf{C}_{2} & \mathbf{K}+\mathbf{C}_{1} & -\mathbf{F A} \mathbf{F}^{\prime} \\
-\mathbf{A}^{\prime} \mathbf{F} & -\mathbf{F}^{\prime} \mathbf{A}^{\prime} \mathbf{F} & \mathbf{K}^{\prime}+\mathbf{C}_{1}^{\prime}
\end{array}\right],
\end{aligned}
$$

where the pertinent matrices are defined as follows:

$$
\begin{aligned}
& M \times M: \quad \mathbf{K}_{m n}=\delta_{m n} \sqrt{\left(k_{F} W / \pi\right)^{2}-n^{2}}, \\
& \left(\mathbf{C}_{1}\right)_{m n}=\delta_{m n} \mathbf{K}_{n n} / \operatorname{tg}\left(\pi \mathbf{K}_{n n} D / W\right), \\
& \left(\mathbf{C}_{2}\right)_{m n}=\delta_{m n} \mathbf{K}_{n n} / \sin \left(\pi \mathbf{K}_{n n} D / W\right), \\
& \mathbf{F}_{m n}=\delta_{m n}(-1)^{n} ; \\
& N \times N: \quad \mathbf{K}_{m n}^{\prime}=\delta_{m n} \sqrt{\left(k_{F} D / \pi\right)^{2}-n^{2}}, \\
& \left(\mathbf{C}_{1}^{\prime}\right)_{m n}=\delta_{m n} \mathbf{K}_{n n}^{\prime} / \operatorname{tg}\left(\pi \mathbf{K}_{n n}^{\prime} W / D\right), \\
& \left(\mathbf{C}_{2}^{\prime}\right)_{m n}=\delta_{m n} \mathbf{K}_{n n}^{\prime} / \sin \left(\pi \mathbf{K}_{n n}^{\prime} W / D\right), \\
& \mathbf{F}_{m n}^{\prime}=\delta_{m n}(-1)^{n} ; \\
& M \times N: \quad \pi \mathbf{A}_{m n}=2 m n /\left[m^{2}-\left(\mathbf{K}_{n n}^{\prime}\right)^{2}\right](W / D)^{3 / 2}, \\
& N \times M: \quad \pi \mathbf{A}_{m n}^{\prime}=2 m n /\left[m^{2}-\left(\mathbf{K}_{n n}\right)^{2}\right](D / W)^{3 / 2} .
\end{aligned}
$$

There are three scattering processes associated with unit $\mathrm{B}$, two are interface scattering processes (described by $\mathbf{S}_{B}^{1}$ and $\mathbf{S}_{B}^{2}$ ) and the other is free propagation in the magnetically defined barrier (described by $\mathbf{S}_{B}^{F}$ ). Therefore, the total scattering matrix $\mathbf{S}_{B}$ in magnetic field region should be the composition of three individual scattering matrices as

$$
\mathbf{S}_{B}=\mathbf{S}_{B}^{1} \otimes \mathbf{S}_{B}^{F} \otimes \mathbf{S}_{B}^{2} .
$$

As unit B is subjected to a magnetic field, the corresponding transverse states have to be calculated first. The vector potentials are chosen as $\mathbf{A}=(0, B x)$ in the finite field zone and $\mathbf{A}=(B y, B x)$ in the zero field region, respectively (see Fig. 3). The longitudinal wave number $k_{m}$ and transverse wave function $\phi_{m}(x)$ satisfy the following equation:

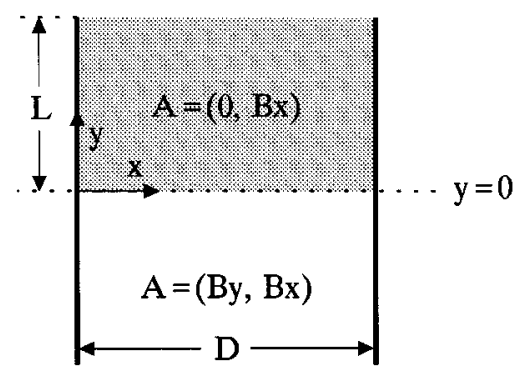

FIG. 3. Schematic illustration of one interface in unit B. The vector potential is continuous across this interface.

$$
\begin{aligned}
& {\left[-\frac{\hbar^{2}}{2 m^{*}} \frac{d^{2}}{d y^{2}}+\frac{m^{*} \omega_{c}^{2}}{2}\left(x+l_{B}^{2} k_{m}\right)^{2}+V(x)\right] \phi_{m}(x)} \\
& =E_{F} \phi_{m}(x),
\end{aligned}
$$

where $\omega_{c}=e B / m^{*}$ is the cyclotron angular frequency, $l_{B}$ $=\sqrt{\hbar / e B}$ is the magnetic length, and $E_{F}$ is the electron Fermi energy. According to the group velocity which is given by

$$
v_{m}=\hbar / m *\left\langle\phi_{m}\left|\left(k_{m}+x / l_{B}^{2}\right)\right| \phi_{m}\right\rangle,
$$

the transverse states can be classified into two categories, the right-moving states $\phi_{m}^{+}$with purely positive real or positive imaginary $v_{m}$ and left-moving states $\phi_{m}^{-}$with purely negative real or negative imaginary $v_{m}$. In our numerical calculations, we expand the transverse wave functions $\phi_{m}^{ \pm}(x)$ in terms of those when $B=0, \varphi_{n}(x)=\sqrt{2 / D} \sin (n \pi x / D)$,

$$
\phi_{m}^{ \pm}(x)=\sum_{n} F_{n m}^{ \pm} \varphi_{n}(x) .
$$

The expansion coefficients $F_{n m}^{ \pm}$'s construct matrix $\mathbf{F}^{ \pm}$. In this article, we use a dimensionless quantity $\beta=\hbar \omega_{c} / E_{1}$ to scale the strength of the magnetic field. Here $E_{1}$ is the eigenenergy of the lowest transverse energy subband in the absence of magnetic field.

After the transverse states have been calculated, $\mathbf{S}_{B}^{F}$ can readily be written as

$$
\mathbf{S}_{B}^{F}=\left[\begin{array}{cc}
\mathbf{0} & \mathbf{P}^{-} \\
\mathbf{P}^{+} & \mathbf{0}
\end{array}\right]
$$

where $\mathbf{P}_{m n}^{ \pm}=\delta_{m n} \exp \left( \pm i k_{m}^{ \pm} L\right)$. We now apply the conventional mode-matching technique to obtain the scattering matrices $\mathbf{S}_{B}^{1}$ and $\mathbf{S}_{B}^{2}$ as

$\mathbf{S}_{B}^{1}=\left[\begin{array}{cc}\mathbf{F}^{+} & -\mathbf{I} \\ \mathbf{F}^{+} \mathbf{K}^{+} & \mathbf{K}-\beta \mathbf{X} / 2\end{array}\right]^{-1}\left[\begin{array}{cc}-\mathbf{F}^{-} & \mathbf{I} \\ -\mathbf{F}^{-} \mathbf{K}^{-} & \mathbf{K}+\beta \mathbf{X} / 2\end{array}\right]$,

$\mathbf{S}_{B}^{2}=\left[\begin{array}{cc}-\mathbf{I} & \mathbf{F}^{+} \\ \mathbf{K}-\beta \mathbf{X} / 2 & \mathbf{F}^{+} \mathbf{K}^{+}\end{array}\right]^{-1}\left[\begin{array}{cc}\mathbf{I} & -\mathbf{F}^{-} \\ \mathbf{K}+\beta \mathbf{X} / 2 & -\mathbf{F}^{-} \mathbf{K}^{-}\end{array}\right]$,

where $\mathbf{I}$ is a unit matrix and

$$
\begin{aligned}
& \mathbf{K}_{m n}=\delta_{m n} \sqrt{\left(k_{F} D / \pi\right)^{2}-m^{2}}, \\
& \mathbf{K}_{m n}^{ \pm}=\delta_{m n} k_{m}^{ \pm} D / \pi, \\
& \mathbf{X}_{m n}=\left\langle\varphi_{m}|\pi x / D| \varphi_{n}\right\rangle .
\end{aligned}
$$

To end this section, we briefly mention one key point in the composition of scattering matrices. In Ref. 21, Tamura and Ando provided the composition law which is suitable to two-terminal scattering regions and can be used to construct the scattering matrix $\mathbf{S}_{B}$ [see Eq. (18)]. While for multipleterminal scattering regions, e.g., units $\mathrm{A}$ and $\mathrm{C}$, the conventional composition law cannot be applied. However, the generalization of the conventional composition law to the multiple-terminal cases is fairly simple. When performing the composition $\mathbf{S}_{C} \otimes \mathbf{S}_{B}$, first we need to regard the threeterminal part, unit $\mathrm{C}$, as a two-terminal section by taking leads 1 and 2 in unit $\mathrm{C}$ as one lead, then we can use the 
conventional composition law as if unit $\mathrm{C}$ is a two-terminal region. After the composition, leads 1 and 2 in unit $C$ become two leads of the compositive unit $C+B$ and lead 2 in unit B becomes the other lead.

\section{NUMERICAL RESULTS}

With the use of the scattering approach described in Sec. II, we calculate the overall scattering-matrix for the system and obtain the corresponding transmission matrix. The numerical results are shown in Fig. 4. Although there are many independent quantities in the transmission matrix, we concentrate ourselves on the wavenumber dependence of two transmission coefficients $T_{14}$ (solid lines) and $T_{23}$ (dotted lines), which reflect the significant scattering properties of the system. In the absence of the magnetic fields, we have $T_{14}=T_{23}$ due to the geometrical symmetry of the structure, which can be found from the panels with $\beta=0$ in Figs. 4(a)4(c). It is also found that the tunneling transmission is relative small for the structures of $D=W$ when $\beta=0$. But for the structure of $D=2 \mathrm{~W}$, the transmission becomes large because the electron transfer is more efficient for a broader coupling region. ${ }^{22}$

When the coupling region is subjected to a magnetic field, the case becomes quite different. From Fig. 4(a) which provides the results for the structure of $D=W$ and $L=0.2 \mathrm{~W}$, it is seen that the behavior of curves $T_{14}$ and $T_{23}$ is very different when $\beta \neq 0$ and the symmetry of the transmission matrix with respect to geometrically symmetrical ports is broken. More specifically, $T_{14}$ becomes larger as increasing the magnetic field while $T_{23}$ is getting smaller, i.e., the magnetic field in the coupling region remarkably enhances the coupling between the two quantum wires for one specific input port while it weakens the coupling for another port. This result can be well understood by the fact that the creation of the edge states located at the hard-wall and the interfaces showing the abrupt changes of the magnetic fields in the coupling region where a magnetic field is applied. The specific moving directions of the electrons in the edge states are indicated by the arrow lines in Fig. 1. When injecting electrons from port 3 , the moving direction of the propagating mode in port 3 is in accordance with that of the upper edge state, thus, they strongly couple to each other. The electrons move along the edge states in the coupling region with a closed orbit, therefore, only a few electrons are transmitted to port 2 in each loop on the closed orbit. On the contrary, when injecting electrons from port 4, the moving direction of the propagating mode in port 4 is opposite to that of the upper edge state, therefore, their coupling cannot take place. In this case, electrons directly tunnel through the magnetic field region to port 1 via the bulk states in the finite field region. Consequently, it makes the transmission coefficient larger and leads to the strong asymmetry, $T_{14}$ larger than $T_{23}$.

The results for the structure with thicker coupling barrier ( $L=1.0 \mathrm{~W})$ are given in Fig. 4(b). The dependence of the transmission on the magnetic field is very similar to that of Fig. 4(a). Different from that of the structure with thin coupling barrier, the transmission spectra exhibit something like those of a band-pass filter, i.e., only those electrons in an en- ergy scope can transmit through the coupling region from the upper wire to the lower one. The other difference lies on the fact that the position of the strongest transmission peak in $T_{23}$ shifts toward the lower wavenumber region as increasing $L$. For the structure with a broader coupling region $(D=2$ W), the results are depicted in Fig. 4(c). As mentioned above, all the transmission coefficients become larger compared with those for the former two structures with narrower width of the coupling region $(D=W)$. For this structure, we want to emphasize the case of $\beta=1.0$. From the panel with $\beta=1.0$, it is clearly seen that $T_{14} \sim 0.5$ within a wide range of the electron wavenumber. Although $T_{24}$ is not plotted in Fig. 4 , it takes a relative small value. Therefore, $T_{14} \sim 0.5$ means $T_{14} \sim T_{34}$. This is one of the most desirable results in designing a good directional coupler because good uniformity of the coupler is required.

\section{DISCUSSIONS AND CONCLUSIONS}

From the results, it is found that the magnetic field has a pronounced effect on the tunneling between the two quantum wires. As the symmetry of the transmission matrix with regard to the geometrically symmetrical ports is broken, the system is expected to be a good quantum directional coupler. For a directional coupler, its performance can be characterized by two major coupler specifications, i.e., the directivity $D$ and uniformity $U$, which are defined as follows:

$$
\begin{aligned}
& D(d B)=10 \log \left(T_{14} / T_{24}\right), \\
& U(d B)=10 \log \left(T_{34} / T_{14}\right),
\end{aligned}
$$

where port 4 is assumed to be the input port. As the quantum coupler considered in this article is expected to be a directional coupler in which the incident electron wave injected into a certain input port (port 4) is transferred to only one or more specified output ports (e.g., ports 3 and 1), the directivity $D$ is one of the most important device specifications. The device is also expected to be a uniformly distributive coupler, it requires that the incident electron wave from the input port is distributed equally among the output ports, so the uniformity $U$ is also considered as another important device specification. A good directional coupler means that it possesses high directivity and good uniformity $(U \sim 0 d B)$. In Fig. 5, we display the calculated coupler specifications for the structure with $D=2 \mathrm{~W}$ and $L=0.2$. Solid curves correspond to $D$ and dotted curves to $U$. From the results, it is evident that the structure under $\beta=1.0$ exhibits high (not the highest) directivity and best uniformity within a wide range of the electron wavenumber.

In conclusion, we have numerically studied the characteristics of an electron waveguide coupler based on two quantum wires coupled each other by a magnetically defined barrier with use of the scattering-matrix method. We have calculated the tunneling transmission spectra for different geometry parameters and magnetic fields. The numerical results can be well understood based on the formation of the edge states in the magnetic field region, and the coupling or decoupling effect between the edge states and the propagating modes in the ports, strongly dependent on their relative moving direction of electrons in these modes. Inspired by the 


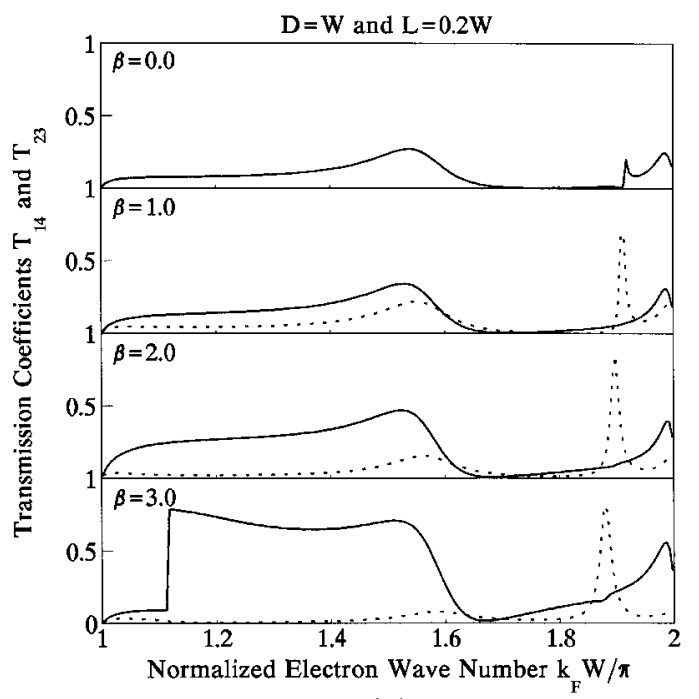

(a)

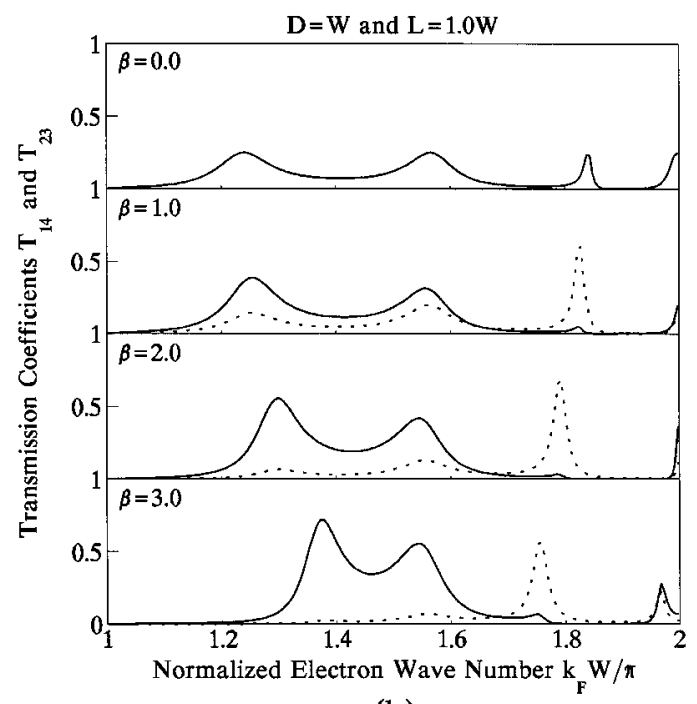

(b)

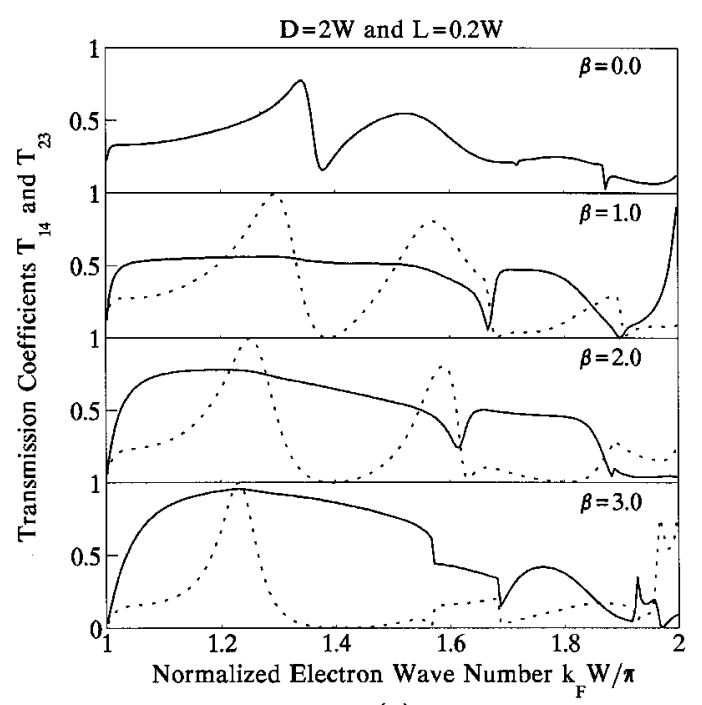

(c)

FIG. 4. Transmission spectrum vs the normalized electron wavenumber $k_{F} W / \pi$ for several values of the strength of the magnetic fields, solid lines for $T_{14}$, dotted lines for $T_{23}$. (a) $D=W, L=0.2 \mathrm{~W}$; (b) $D=W$ and $L$ $=1.0 \mathrm{~W}$; and (c) $D=2 \mathrm{~W}, L=0.2 \mathrm{~W}$.

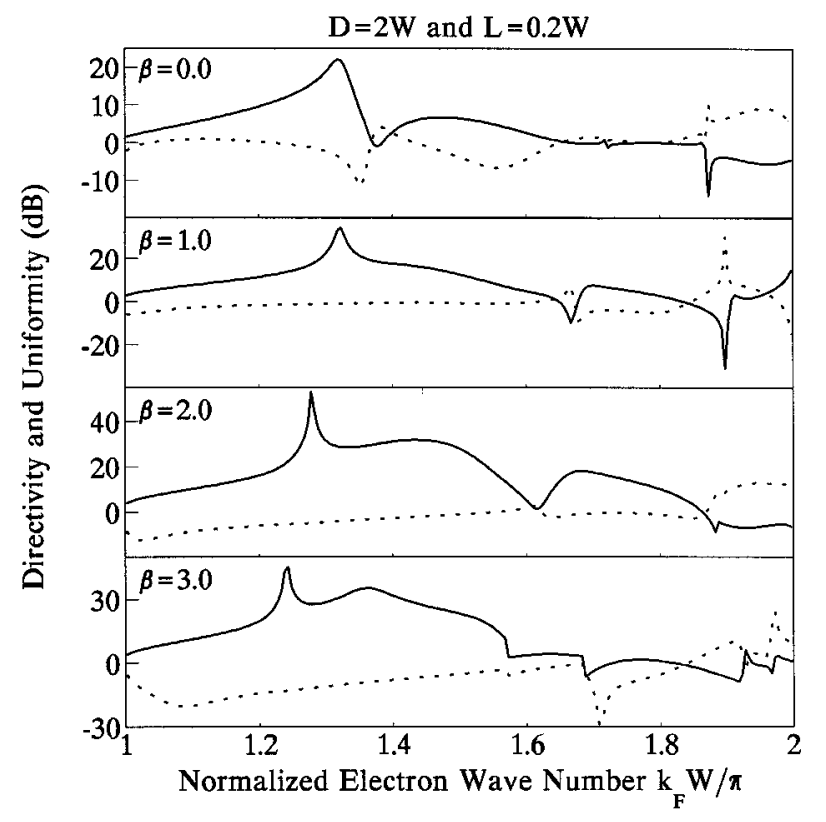

FIG. 5. Coupler specifications (the directivity and uniformity) vs the normalized electron wavenumber $k_{F} W / \pi$ for several values of the strength of the magnetic fields, solid lines for the directivity and dotted lines for the uniformity.

strong asymmetry of transmission, it is expected that the magnetically coupled quantum wires may be a good candidate of the quantum directional coupler.

\section{ACKNOWLEDGMENTS}

The authors would thank Dr. X. H. Wang for the valuable discussions. They gratefully acknowledge the support by a research grant from the Croucher Foundation, a RGC grant from the Government of Hong Kong under Grant No. HKU 261/95P, and a research grant from the Chinese National Science Foundation. They also thank the Computer Center of the University of Hong Kong for computational facilities.

${ }^{1}$ B. J. van Wees, H. van Houten, C. W. J. Beenakker, J. G. Williamson, L. P. Kouwenhoven, D. Van der Marel, and C. T. Foxon, Phys. Rev. Lett. 60, 848 (1988).

${ }^{2}$ D. A. Wharam, M. Pepper, H. Ahmed, J. E. F. Frost, D. G. Hasko, D. C. Peacock, D. A. Ritchie, and G. A. C. Jones, J. Phys.: Condens. Matter 21, L209 (1988).

${ }^{3}$ F. A. Buot, Phys. Rep. 234, 73 (1993).

${ }^{4}$ F. Sols, M. Macucci, U. Ravaioli, and K. Hess, Appl. Phys. Lett. 54, 350 (1989).

${ }^{5}$ J. Appenzeller, Ch. Schroer, Th. Schäpers, A. v. d. Hart, A. Förster, B. Lengeler, and H. Lüth, Phys. Rev. B 53, 9959 (1996).

${ }^{6}$ J. A. del Alamo and C. C. Eugster, Appl. Phys. Lett. 56, 78 (1990).

${ }^{7}$ C. C. Eugster and J. A. del Alamo, Phys. Rev. Lett. 67, 3586 (1991).

${ }^{8}$ J. Wang, Y. J. Wang, and H. Guo, Phys. Rev. B 46, 2420 (1992).

${ }^{9}$ M. Macucci, A. Galick, and U. Ravaioli, Phys. Rev. B 52, 5210 (1995).

${ }^{10}$ O. Vanbésien and D. Lippens, Appl. Phys. Lett. 65, 2439 (1994).

${ }^{11}$ O. Vanbésien and D. Lippens, Phys. Rev. B 52, 5144 (1995).

${ }^{12}$ B. L. Altshuler and L. B. Ioffe, Phys. Rev. Lett. 69, 2979 (1992); T. Sugiyama and N. Nagoasa, ibid. 70, 1980 (1993); S.-C. Zhang and D. P. Arovas, ibid. 72, 1886 (1994).

${ }^{13}$ Z. L. Ji and D. W. L. Sprung, Phys. Rev. B 54, 8044 (1996).

${ }^{14}$ Y. Takagaki and K. Ploog, Phys. Rev. B 51, 7017 (1995).

${ }^{15}$ Y. Takagaki and K. Ploog, Phys. Rev. B 53, 3885 (1996). 
${ }^{16}$ H. Xu, Phys. Rev. B 52, 5803 (1995).

${ }^{17}$ C. S. Kim and O. Olendski, Phys. Rev. B 53, 12917 (1996).

${ }^{18}$ J. R. Shi and B. Y. Gu, Phys. Rev. B 55, 9941 (1997).

${ }^{19}$ W. D. Sheng and J. B. Xia, Phys. Lett. A 220, 268 (1996).
${ }^{20}$ W. D. Sheng, J. Appl. Phys. (unpublished).

${ }^{21}$ H. Tamura and T. Ando, Phys. Rev. B 44, 1792 (1991).

${ }^{22}$ W. D. Sheng and J. B. Xia, Appl. Phys. A: Solids Surf. 64, 167 (1996); W. D. Sheng, J. Phys. D 29, 3166 (1996). 